\title{
Angle-dependent time delay in two-color XUV+IR photoemission of He and Ne
}

\author{
I. A. Ivanov ${ }^{1,2}$ and A. S. Kheifets ${ }^{1}$ \\ ${ }^{1}$ Research School of Physics and Engineering, The Australian National University, Canberra ACT 0200, Australia \\ ${ }^{2}$ Center for Relativistic Laser Science, Institute for Basic Science (IBS), Gwangju 500-712, Republic of Korea
}

(Received 20 April 2017; published 11 July 2017)

\begin{abstract}
We solve the time-dependent Schrödinger equation for a noble gas atom ( $\mathrm{He}$ and $\mathrm{Ne}$ ) driven by an ionizing XUV and dressing IR fields. From this solution we deduce an angular dependence of the photoemission time delay as measured by the RABBITT (reconstruction of attosecond beating by interference of two-photon transitions) technique. We use a recent angle-resolved RABBITT measurement on helium [S. Heuser et al., Phys. Rev. A 94, 063409 (2016)] to test and calibrate our theoretical model. Based on this calibration, we find no significant difference between the time delay in He measured in the angle-integrated RABBITT experiments [C. Palatchi et al., J. Phys. B 47, 245003 (2014) and D. Guénot et al., ibid. 47, 245602 (2014)] and measured or calculated in the polarization axis direction. The angular dependence of the photoemission time delay of $\mathrm{Ne}$ is shown to be qualitatively different from He because of the different orbital character of the valence $2 p$ orbital. The angular momentum projection dependence of the time delay in $\mathrm{Ne}$ is also investigated.
\end{abstract}

DOI: 10.1103/PhysRevA.96.013408

\section{INTRODUCTION}

A measurable time delay in laser-driven atomic ionization has been discovered recently [1,2]. Since the first pioneering experiments, the time-delay spectroscopy of laser-induced atomic ionization (attosecond chronoscopy) has become a rapidly developing field [3]. In a recent report, Heuser et al. [4] investigated an angular dependence of the photoemission time delay in helium as measured by the RABBITT (reconstruction of attosecond beating by interference of two-photon transitions) technique. The RABBITT technique builds on the interference of two ionization processes leading to the same photoelectron state by (i) absorption of $\omega_{2 q-1}$ and $\omega$ or (ii) absorption of $\omega_{2 q+1}$ and stimulated emission of $\omega$. Both ionization processes lead to the appearance of a sideband (SB) of the order of $2 q$ in between the one-photon harmonic peaks in the photoelectron spectrum. The sideband magnitude oscillates with the relative phase between the XUV and IR pulses $[5,6]$

$$
S_{2 q}(\tau)=A+B \cos [2 \omega \tau-C], \quad C=\Delta \phi_{2 q}+\Delta \theta_{2 q},
$$

where $\tau=\varphi / \omega$ denotes the phase delay of the IR field. The term $\Delta \phi_{2 q}=\phi_{2 q+1}-\phi_{2 q-1}$ denotes the phase difference between two neighboring odd harmonics $2 q \pm 1$ that is related to the finite-difference group delay of the attosecond pulse as $\tau_{2 q}^{(\mathrm{GD})}=\Delta \phi_{2 q} / 2 \omega$. The additional term $\Delta \theta_{2 q}=\theta_{2 q+1}^{(-)}-\theta_{2 q-1}^{(+)}$ arises from the phase difference of the atomic ionization amplitude for emission (-) and absorption (+) paths, respectively. This phase difference can be converted to the atomic delay

$$
\tau_{a}=\Delta \theta_{2 q} / 2 \omega=\tau_{W}+\tau_{\mathrm{CC}},
$$

which contains the two distinct components [7]. Here $\tau_{W}$ is the Wigner-like time delay associated with the XUV absorption and $\tau_{\mathrm{CC}}$ is a correction due to the continuum-continuum (CC) transitions in the IR field. The latter term, $\tau_{\mathrm{CC}}$, can also be understood as a coupling of the long-range Coulomb ionic potential and the laser field in the context of streaking [8,9].

In the case of helium with only one $1 s \rightarrow E p$ photoemission channel, the Wigner time delay $\tau_{W}$ does not depend on the photoelectron detection angle relative to the polarization vector. The early investigations of the $\tau_{\mathrm{CC}}$ correction [7] showed no dependence over various angular momentum paths in hydrogen, e.g., the transitions $s \rightarrow p \rightarrow s$ and $s \rightarrow p \rightarrow d$ showed $\tau_{\mathrm{CC}}$ in excellent agreement. This would imply that the RABBITT measured time delay in He should be angle independent. This assumption was challenged in a recent experiment by Heuser et al. [4] in which the RABBITT technique was supplemented with the COLTRIMS (cold target recoil ion momentum spectroscopy) apparatus. This combination made it possible to relate the time delay to a specific photoelectron detection angle relative to the joint polarization axis of the XUV and IR pulses. The finding of Heuser et al. [4] is significant because the helium atom is often used as a convenient reference to determine the time delay in other target atoms. If the RABBITT measurement is not angle resolved, like in the experiments by Palatchi et al. [10] and Guénot et al. [11], the angular dependence of the time delay in the reference atom may compromise the accuracy of the time-delay determination in other target atoms. However, in the present work, we demonstrate that the magnitude of the RABBITT signal drops off very rapidly as $\cos ^{4} \theta$ where $\theta$ is the angle relative to the polarization direction and hence the angle-integrated RABBITT measurement on He would return the time delay very close to that calculated or measured in the polarization direction.

In the case of $\mathrm{Ne}$ and heavier noble gases, both components of the measured time delay $\tau_{a}=\tau_{W}+\tau_{\mathrm{CC}}$ are angle dependent. Indeed, for a one-photon transition from a bound $n p$ orbital, there are two competing $s$ and $d$ continua which can lead to the angle-dependent Wigner time delay $\tau_{W}$ [12-14]. Because of the propensity rule [15], the $d$ channel is strongly dominant except in the vicinity of its Cooper minimum. Such a minimum is absent in Ne. So the Wigner time delay should depend on the emission angle very weakly. Therefore any measurable angular variation of the time delay in Ne should be attributed almost entirely to the $\tau_{\mathrm{CC}}$ component. The latter arises due to the competition of the CC transitions $d \rightarrow p$ or $d \rightarrow f$. This competition is analyzed in the present work. 


\section{THEORY AND NUMERICAL IMPLEMENTATION}

We solve the time-dependent Schrödinger equation (TDSE) for a target atom described within the single active electron (SAE) approximation

$$
i \partial \Psi(\boldsymbol{r}) / \partial t=\left[\hat{H}_{\text {atom }}+\hat{H}_{\text {int }}(t)\right] \Psi(\boldsymbol{r}),
$$

where $\hat{H}_{\text {atom }}$ is the Hamiltonian of the field-free atom with an effective one-electron potential [16]. The Hamiltonian $\hat{H}_{\text {int }}(t)$ describes the interaction with the external field and is written in the velocity gauge as

$$
\hat{H}_{\mathrm{int}}(t)=\boldsymbol{A}(t) \cdot \hat{\boldsymbol{p}}, \quad \boldsymbol{A}(t)=-\int_{0}^{t} \boldsymbol{E}\left(t^{\prime}\right) d t^{\prime} .
$$

As compared to the alternative length gauge, this form of the interaction has a numerical advantage of a faster convergence.

The vector potential of the APT is modeled as the sum of 11 Gaussian pulses of altering polarity shifted by half of the IR period $T=2 \pi / \omega$ :

$$
\begin{aligned}
A_{x}(t)= & \sum_{n=-5}^{5}(-1)^{n} A_{n} \exp \left(-2 \ln 2 \frac{(t-n T / 2)^{2}}{\tau_{x}^{2}}\right) \\
& \times \cos \left[\omega_{x}(t-n T / 2)\right] .
\end{aligned}
$$

The amplitude of each pulse is defined as

$$
A_{n}=A_{0} \exp \left(-2 \ln 2 \frac{(n T / 2)^{2}}{\tau_{T}^{2}}\right)
$$

where $A_{0}$ is the vector potential amplitude related to the field intensity $I=\left(\omega^{2} / c^{2}\right) A_{0}^{2}$. The XUV central frequency is $\omega_{x}=$ 1.378 a.u. $=37.5 \mathrm{eV}$. The time constants $\tau_{x}=0.14 \mathrm{fs}$ and $\tau_{T}=4.83$ fs determine the length of an XUV pulse and the attosecond pulse train (APT), respectively. The field intensity of the APT is chosen at $5 \times 10^{8} \mathrm{~W} / \mathrm{cm}^{2}$ and the XUV frequency $\omega_{x} \simeq 25 \omega$.

The vector potential of the IR pulse is modeled by the cosine-squared envelope

$$
A(t)=A_{0} \cos ^{2}\left(\frac{\pi(t-\tau)}{2 \tau_{\mathrm{IR}}}\right) \cos [\omega(t-\tau)],
$$

with an intensity of $3 \times 10^{11} \mathrm{~W} / \mathrm{cm}^{2}$ and pulse duration of $\tau_{\mathrm{IR}}=14.5 \mathrm{fs}$. The IR pulse is shifted relative to the APT by a variable delay $0 \leqslant \tau \leqslant 0.5 T$. A positive delay, $\tau>0$, corresponds to the IR pulse being delayed with respect to the center of the XUV pulse train. Further, the laser photon energy is $\omega=0.05841$ a.u. $=1.59 \mathrm{eV}$, which corresponds to a period of $T=2 \pi / \omega=107$ a.u. $=2.60$ fs. The laser pulse duration is $\tau=5.58 T=14.5 \mathrm{fs}$.

To solve the TDSE, we follow the strategy tested in our previous works $[17,18]$. The solution of the TDSE is presented as a partial-wave series

$$
\Psi(\boldsymbol{r}, t)=\sum_{\substack{l=0 \\|m| \leqslant l}}^{L_{\max }} f_{l}(r, t) Y_{l m}(\theta, \phi)
$$

with only $m=m_{0}$ momentum projections retained for the linearly polarized light. Here $m_{0}$ refers to the target orbital: $m_{0}=0$ for $\mathrm{He} 1 s$ and $m_{0}=0, \pm 1$ for $\mathrm{Ne} 2 p$. The radial part of the TDSE is discretized on the grid with the step size $\delta r=0.05$ a.u. in a box of the size $R_{\max }=2000$ a.u. The number of partial waves in Eq. (7) was limited to $L_{\max }=5$ which ensured convergence in the velocity gauge calculations.

Substitution of the expansion (7) into the TDSE gives a system of coupled equations for the radial functions $f_{l \mu}(r, t)$, describing evolution of the system in time. To solve this system, we use the matrix iteration method [19]. The ionization amplitudes $a(\boldsymbol{k})$ are obtained by projecting the solution of the TDSE at the end of the laser pulse on the set of the ingoing scattering states of the target Hamiltonian. Squared amplitudes $|a(\boldsymbol{k})|^{2}$ give the photoelectron spectrum in a given direction $\hat{\boldsymbol{k}}$ determined by the azimuthal angle $\theta_{k}$.

After collecting the photoelectron spectra from the TDSE in a given direction, the SB intensity oscillation with the variable time delay between the APT and IR fields is fitted with the cosine function (1) using the nonlinear Marquardt-Levenberg algorithm. The quality of the fit is very good with the errors in all three parameters not exceeding $1 \%$.

\section{RESULTS}

\section{A. Helium}

The angular dependence of the parameters $A, B$, and $C$ in Eq. (1) for SB 20 is shown in Fig. 1. It follows from the soft photon approximation (SPA) [20] that the angular dependence of the $A=\left|\mathcal{M}_{\boldsymbol{k}}^{(-)}\right|^{2}+\left|\mathcal{M}_{\boldsymbol{k}}^{*(+)}\right|^{2}$ and $B=2 \operatorname{Re}\left[\mathcal{M}_{\boldsymbol{k}}^{(-)} \mathcal{M}_{\boldsymbol{k}}^{*(+)}\right]$ parameters are simple $\cos ^{4} \theta$ functions for an initial $s$ state,

$$
A, B \propto\left|J_{1}\left(\boldsymbol{\alpha}_{0} \cdot \boldsymbol{k}\right)\right|^{2}|\langle f|z| i\rangle|^{2} \propto \cos ^{4} \theta_{k} .
$$
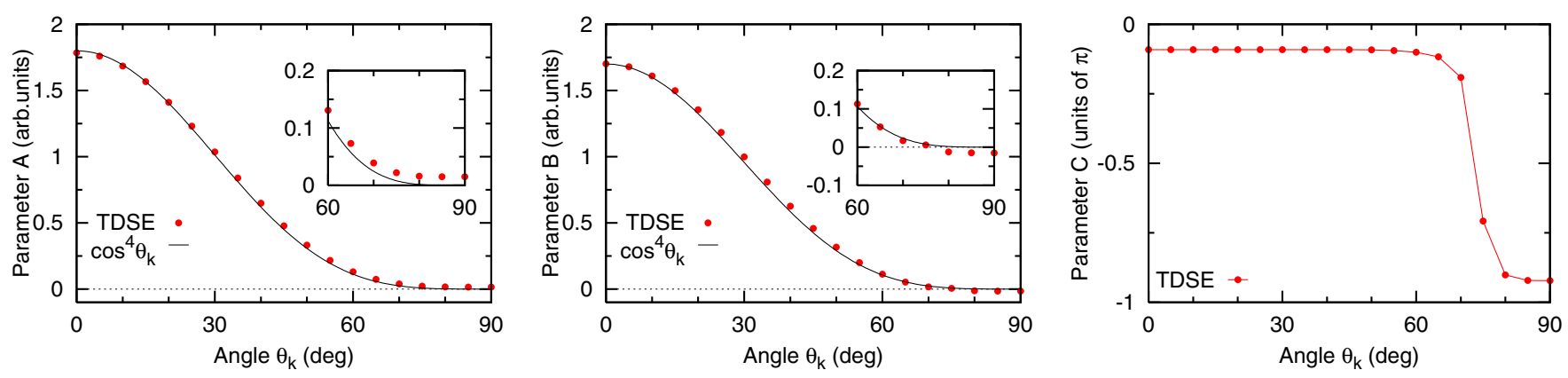

FIG. 1. Angular dependence of the fitting parameters $A, B$, and $C$ for SB 20 in He as calculated by the TDSE. The insets show the variation of the $A$ and $B$ parameters near $90^{\circ}$. The $\cos ^{4} \theta_{k}$ fit to the $A$ and $B$ parameters is also displayed. 
TABLE I. Atomic time delay $\tau_{a}$ and its various components $\tau_{W}$ and $\tau_{\mathrm{CC}}$ in the $\hat{z}$ direction for various sidebands. Under $\tau_{\mathrm{CC}}$, (i) is the atomic delay minus Wigner delay, and (ii) is the fit to exact hydrogen calculation [7].

\begin{tabular}{ccccccc}
\hline \hline SB & $n \omega$ & $E$ & $\tau_{a}($ as) & $\tau_{W}($ as $)$ & \multicolumn{2}{c}{$\tau_{\mathrm{CC}}($ as $)$} \\
$n$ & $\mathrm{eV}$ & $\mathrm{eV}$ & $\mathrm{TDSE}$ & $\mathrm{RPAE}$ & (i) & (ii) \\
\hline 18 & 27.9 & 3.3 & -85 & 231 & -316 & -315 \\
20 & 31.0 & 6.4 & -61 & 60 & -121 & -129 \\
22 & 34.1 & 9.5 & -46 & 30 & -76 & -83 \\
24 & 37.2 & 12.6 & -37 & 16 & -53 & -57 \\
\hline \hline
\end{tabular}

Here $\mathcal{M}_{k}^{( \pm)}$are complex amplitudes for angle-resolved photoelectrons produced by adding or subtracting an IR photon, respectively. In Eq. (8) we made a linear approximation to the Bessel function as the parameter $\alpha_{0}=F_{0} / \omega^{2}$ is small in a weak IR field. This simple dependence fits very well both the $A$ and $B$ parameters. The only deviation occurs at large angles where the $B$ parameter becomes negative while $\cos ^{4} \theta$ always remains positive (see insets of Fig. 1). The SPA also predicts no angular variation of the time delay. So angle-dependent time delay and alteration of sign of the $B$ parameter are both signs of breakdown of the SPA.

The group delay $\tau_{\mathrm{GD}}$ of the APT is zero in our approach since we consider Fourier limited attosecond pulses by setting $\phi_{2 q+1}=0$, for all integers $q$ in the frequency comb. Hence the parameter $C$ can be converted directly into the atomic time delay as $\tau_{a}=C / 2 \omega$ according to Eq. (1). The atomic time delay obtained in this fashion is given in Table I for the direction along the polarization axis, which we refer to as the zero angle for photoemission. To connect with Eq. (2) we also show the breakdown of the atomic delay into the Wigner time delay $\tau_{W}$, which was computed separately by the one-photon random phase approximation with exchange (RPAE) [21], and the extracted continuum-continuum delay $\tau_{\mathrm{CC}}$ which we compare with an earlier calculation [7]. The discrepancy between the two $\mathrm{CC}$ quantities is reasonably small, less than 10 as, even though the present result is obtained for He whereas the calculation [7] was performed for $\mathrm{H}$. This reflects a universal nature of the CC correction which is not sensitive to the target $n s$ orbital. The variation of the atomic time delay relative to the zero angle polarization direction $\Delta \tau=\tau_{a}\left(\theta_{k}\right)-\tau\left(0^{\circ}\right)$ is displayed in Fig. 2 for SBs 18-24. In the same figure, we plot the experimental data and the lowest order perturbation theory (LOPT) calculation from Heuser et al. [4].

In the experimentally accessible angular range of $0-65^{\circ}$, the angular variation of the time delay is rather small. It progressively decreases from about 60 as in SB 18 to less than 30 as in SB 24. It is expected that for higher sidebands it will be even smaller as the CC phases for the $s$ and $d$ waves become indistinguishable in the high photon energy limit. Given the rapid drop of the magnitude $A$ and $B$ parameters in Eq. (1) with the detection angle as $\cos ^{4} \theta_{k}$, the angle-averaged time delay $\bar{\tau}_{a}$ will be very close to that recorded in the polarization direction of light at the zero degree angle $\tau_{a}(\theta=0)$. This allows the use of the helium atom as a convenient standard both in the angle-specific streaking and angle-averaged RABBITT time-delay measurements.

\section{B. Neon}

The angular dependence of the parameters $A, B$, and $C$ in $\mathrm{Ne}$ for SB 20 is shown in Fig. 3. This variation is qualitatively similar to that in $\mathrm{He}$ as displayed in Fig. 1. However, unlike in He, where the $\cos ^{4} \theta_{k}$ dependence fits both the $A$ and $B$ parameters very well, in $\mathrm{Ne}$ this dependence deviates noticeably from the TDSE calculation. This is so because the squared dipole matrix element $|\langle f|z| i\rangle|^{2} \propto 1+\beta P_{2}\left(\cos \theta_{k}\right)$ deviates from $\cos ^{2} \theta_{k}$ for $\beta \neq 2$ which is the case of the $\mathrm{Ne}$ $2 p$ shell where for SB $20 \beta \approx 0.3$ [22]. In this case a more accurate fit is provided by the second Legendre polynomial expression as shown in Fig. 3.

Even though the $A$ and $B$ parameters decrease rather quickly with the detection angle, this decrease is not as rapid in $\mathrm{Ne}$ as prescribed by the soft photon approximation. The $C$ parameters both in $\mathrm{Ne}$ and $\mathrm{He}$ experience the drop of one unit of $\pi$. However, in Ne, this drop is more gradual than in $\mathrm{He}$.

Angular variations of time delay in neon for SBs 18-24 are shown in Fig. 4. The analogous set of He data is also plotted for the sake of comparison. The angular dependence of the time delay in $\mathrm{Ne}$ is more pronounced at small angles where the $\mathrm{He}$ data are virtually flat. The flattening of the angular dependence in $\mathrm{Ne}$ is also happening toward the higher order sidebands, but not as quickly as in He.

As mentioned in the Introduction, both components of the atomic time delay in $\mathrm{Ne}$ can be angle dependent. However, because of the lack of the Cooper minimum, the angular dependence of the Wigner time delay $\tau_{W}$ should be weak. This dependence is shown in Fig. 4 in comparison with
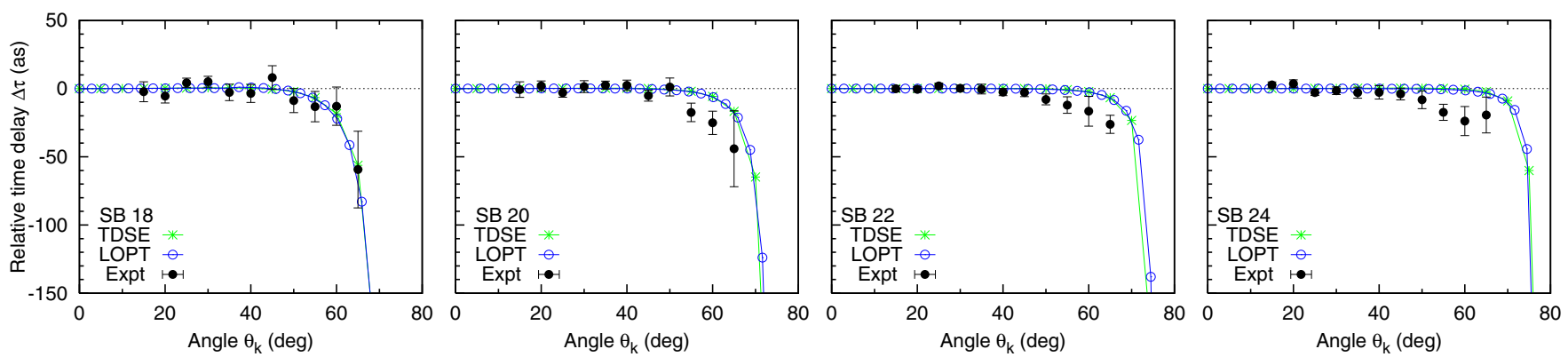

FIG. 2. Variation of the time delay relative to the zero angle $\Delta \tau_{a}=\tau_{a}\left(\theta_{k}\right)-\tau_{a}\left(0^{\circ}\right)$ for SBs 18,20 , 22, and 24 . The TDSE results, LOPT calculations, and experimental data from Heuser et al. [4] are shown. 

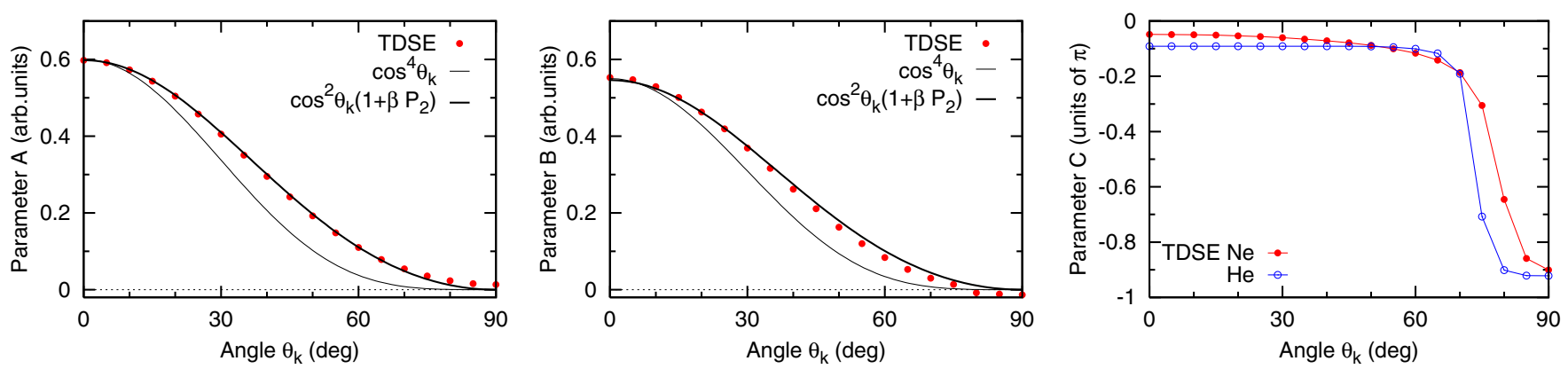

FIG. 3. Angular dependence of the fitting parameters $A, B$, and $C$ for SB 20 in Ne showing the TDSE calculation, the $\cos ^{4} \theta_{k}$ fit to the $A$ and $B$ parameters, and the fit with actual anisotropy parameter $\beta=0.3$. The TDSE result for He is also shown for parameter $C$.

the angular variation of the total atomic time delay $\tau_{a}$. The Wigner time delay, calculated in the relativistic random-phase approximation [14] varies noticeably only in the lowest sideband and this variation is significantly smaller that that of the total time delay in all the sidebands. This indicates that the angular variation of the measured time delay in $\mathrm{Ne}$ should be attributed almost solely to the $\tau_{\mathrm{CC}}$ component. The latter arises due to the competition of the CC transitions $d \rightarrow p$ or $d \rightarrow f$. Unlike in helium, where the two CC transitions compete strongly only beyond the magic angle, this competition seems to be noticeable in $\mathrm{Ne}$ even at small angles.

To quantify this competition, we consider the case of the $m=0$ projection in the target orbital and adapt the parametrization of the ionization amplitudes similar to that suggested in Heuser et al. [4]:

$$
\mathcal{M}_{\boldsymbol{k}}^{( \pm)} \propto Y_{10}(\boldsymbol{n})+c_{f p}^{( \pm)} e^{i \phi_{f p}^{( \pm)}} Y_{30}(\boldsymbol{n}),
$$

where $c_{f p}^{( \pm)}$and $\phi_{f p}^{( \pm)}$are the magnitude ratio and phase difference between the $f$ and $p$ partial-wave components of the absorption (+) and emission (-) amplitudes, respectively. Fitting the angular dependence of the time delay in the SB 18 for $m=0$ is shown in Fig. 5. The values of the fitting parameters are $c_{f p}^{ \pm}=0.39,1.68$ and $\phi_{f p}^{ \pm}=1.65,0.54$. In comparison in SB 20 of He, $c_{d s}^{ \pm}=0.67,1.17$ and $\phi_{d s}^{ \pm}=0.082,0.076$. In the high energy limit of $\mathrm{He}, c_{d s}^{+}=1 / c_{d s}^{-}=\sqrt{4 / 5} \approx 0.89$ whereas for Ne this limit is $\sqrt{4 / 7} \approx 0.75$. The case of Ne seems to be farther away from the asymptotic limit in which no angular dependence should be observed. Even more contrasting is the phase difference in $\mathrm{Ne}$ which is an order of magnitude larger than in He.
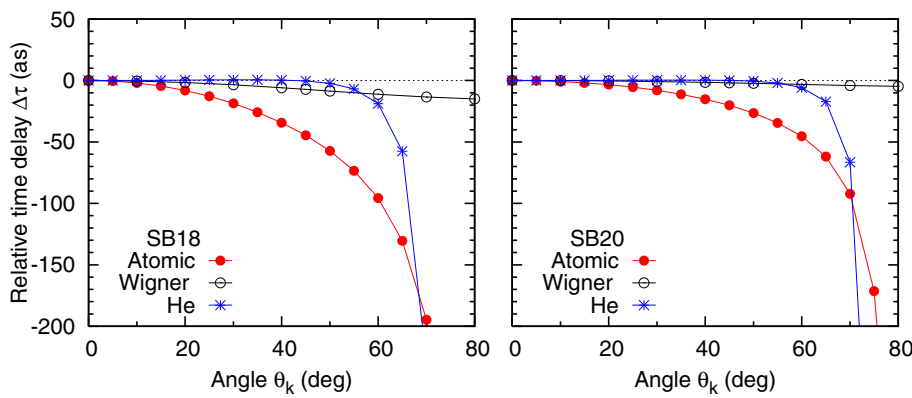

FIG. 4. Variation of the atomic time delay relative to the zero emission angle $\Delta \tau_{a}=\tau_{a}\left(\theta_{k}\right)-\tau_{a}\left(0^{\circ}\right)$ for SBs $18,20,22$, and 24 in neon. Shown are the TDSE results labeled "Atomic," the analogous calculation for He, and the angular variation of the Wigner time delay $\Delta \tau_{W}=\tau_{W}\left(\theta_{k}\right)-\tau_{W}\left(0^{\circ}\right)$ in one-photon ionization.
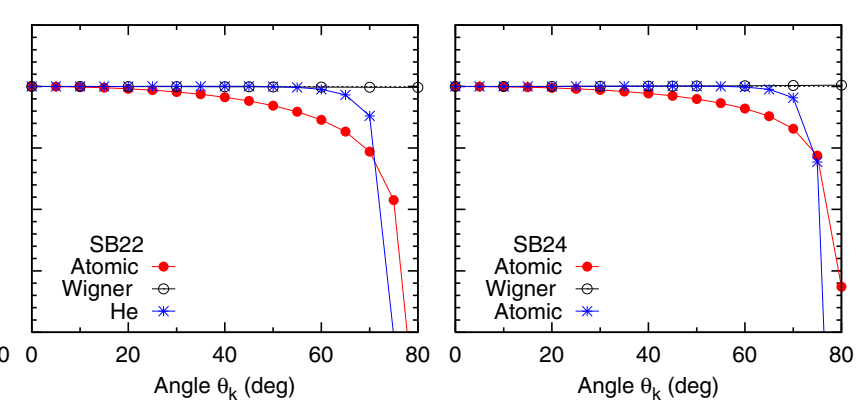

Quite remarkably, the $m=0$ angular dependence of the atomic time delay is very different from that summed over all the $m=$ projection. It is dominated by the sharp drop near the kinematic node of the $Y_{30}$ spherical harmonic. This drop all but disappears in the angular dependence summed over all the $m$ projections because $Y_{3 \pm 1}$ fill the node. Despite this difference, the parametrization (9) is still meaningful to quantify the competition of the CC transitions $d \rightarrow f$ and $d \rightarrow p$ because their strengths depend only on the radial integrals.

\section{CONCLUSION}

In the present work we studied angular variation of the atomic time delay in the RABBIT measurement on helium and neon. We employed a numerical solution of the time-dependent Schrödinger equation for a single atomic electron driven by a combination of the XUV and IR fields with parameters similar to those used in a recent RABBITT measurement by Heuser et al. [4]. Our results for He compare favorably with this measurement. Based on this agreement, we make further predictions for neon which is yet to be measured in an angle-resolved RABBITT experiment.

In comparison to $\mathrm{He}$, the angular variation of time delay in $\mathrm{Ne}$ is more pronounced at small ejection angles relative to the polarization axis where the He time delay is essentially flat. This can be explained by a larger phase difference between the $d \rightarrow f$ and $d \rightarrow p$ CC transitions in the absorption and emission channels. The analogous phase difference between the $p \rightarrow d$ and $p \rightarrow s$ CC transitions in $\mathrm{He}$ is an order of magnitude smaller. 


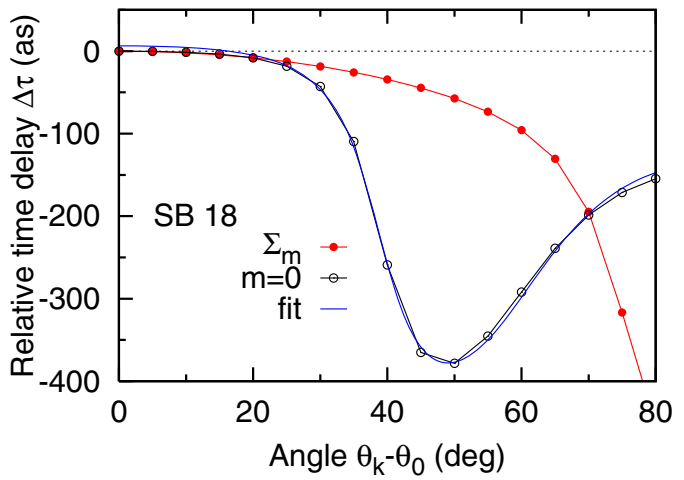

FIG. 5. Angle-dependent time delay in neon for SB 18. The full TDSE calculation summed over all the $m$ projections is shown by the (red) filled circles whereas the $m=0$ calculation is visualized by the open circles. Parametric fit to the latter calculation with Eq. (9) is shown by the blue solid line.

We also analyze the magnitude of the RABBITT signal in both targets and find it dropping off very rapidly away from the polarization direction. In He, the RABBITT signal drops as $\cos ^{4} \theta_{k}$, much faster than the usual dipole factor $\cos ^{2} \theta_{k}$. The difference between these two factors becomes particularly noticeable at large ejection angles: at $\theta_{k}=70^{\circ}$ the one-photon angular factor leaves $\sim 10 \%$ magnitude relative to the maximum whereas the two-photon factor leaves only $\sim 1 \%$. It is for this reason that Heuser et al. [4] were not able to extend their measurements beyond the ejection angle of $65^{\circ}$. However, for the same reason, the angle-integrated RABBITT measurements on $\mathrm{He}$ and $\mathrm{Ne}$ in $[10,11]$ could be compared directly with the time delay calculated along the polarization axis. In contrast, the angular variation of the atomic time delay in Ar was found noticeable in [10] as the angle-averaged calculations were found much closer to the experiment than that in the polarization direction.

The angular dependence of the RABBITT time delay in molecules becomes even more pronounced because of an additional anisotropy relative to the molecular axis. This can be seen from a recent theoretical study on the hydrogen molecular ion [23]. Angle-integrated RABBITT experiments have been reported on other molecules [24] and work is underway to make this measurement angle resolved. So extension of the theory to complex molecules is warranted.

\section{ACKNOWLEDGMENTS}

The authors are grateful to L. Argenti, Á. H. Galán, F. Martín, S. Heuser, C. Cirelli, U. Keller, M. Dahlström, and E. Lindroth for many stimulating discussions. We acknowledge support by the Australian Research Council in the form of the Discovery Grant No. DP120101805. I.A. acknowledges support from the Institute for Basic Science, Gwangju, Republic of Korea, under IBS-R012-D1. Resources of the National Computational Infrastructure (NCI Australia) facility were employed.
[1] M. Schultze et al., Science 328, 1658 (2010).

[2] K. Klünder, J. M. Dahlström, M. Gisselbrecht, T. Fordell, M. Swoboda, D. Guénot, P. Johnsson, J. Caillat, J. Mauritsson, A. Maquet, R. Taïeb, and A. L'Huillier, Phys. Rev. Lett. 106, 143002 (2011).

[3] R. Pazourek, S. Nagele, and J. Burgdörfer, Rev. Mod. Phys. 87, 765 (2015).

[4] S. Heuser, A. J. Galán, C. Cirelli, C. Marante, M. Sabbar, R. Boge, M. Lucchini, L. Gallmann, I. Ivanov, A. S. Kheifets et al., Phys. Rev. A 94, 063409 (2016).

[5] H. Muller, Appl. Phys. B 74, s17 (2002).

[6] E. S. Toma and H. G. Muller, J. Phys. B 35, 3435 (2002).

[7] J. M. Dahlström et al., Chem. Phys. 414, 53 (2013).

[8] C.-H. Zhang and U. Thumm, Phys. Rev. A 84, 033401 (2011).

[9] R. Pazourek, S. Nagele, and J. Burgdorfer, Faraday Discuss. 163, 353 (2013).

[10] C. Palatchi et al., J. Phys. B 47, 245003 (2014).

[11] D. Guénot et al., J. Phys. B 47, 245602 (2014).
[12] J. M. Dahlström and E. Lindroth, J. Phys. B 47, 124012 (2014).

[13] J. Wätzel, A. S. Moskalenko, Y. Pavlyukh, and J. Berakdar, J. Phys. B 48, 025602 (2015).

[14] A. Kheifets, A. Mandal, P. C. Deshmukh, V. K. Dolmatov, D. A. Keating, and S. T. Manson, Phys. Rev. A 94, 013423 (2016).

[15] U. Fano, Phys. Rev. A 32, 617 (1985).

[16] A. Sarsa, F. J. Gálvez, and E. Buendia, At. Data Nucl. Data Tables 88, 163 (2004).

[17] I. A. Ivanov, Phys. Rev. A 83, 023421 (2011).

[18] I. A. Ivanov and A. S. Kheifets, Phys. Rev. A 87, 033407 (2013).

[19] M. Nurhuda and F. H. M. Faisal, Phys. Rev. A 60, 3125 (1999).

[20] A. Maquet and R. Taïeb, J. Mod. Opt. 54, 1847 (2007).

[21] A. S. Kheifets, Phys. Rev. A 87, 063404 (2013).

[22] K. Codling, R. G. Houlgate, J. B. West, and P. R. Woodruff, J. Phys. B 9, L83 (1976).

[23] V. V. Serov and A. S. Kheifets, Phys. Rev. A 93, 063417 (2016).

[24] M. Huppert, I. Jordan, D. Baykusheva, A. von Conta, and H. J. Wörner, Phys. Rev. Lett. 117, 093001 (2016). 